Manuscript prepared for the

Proceedings of the $24^{\text {th }}$ International Symposium on Discharges and Electrical Insulation in Vacuum

(ISDEIV)

Braunschweig, Germany, August 30-Sept 3, 2010

\title{
Ion Species and Charge States of Vacuum Arc Plasma with Gas Feed and Longitudinal Magnetic Field
}

\author{
Efim Oks ${ }^{1}$ and André Anders ${ }^{2}$ \\ ${ }^{1}$ High Current Electronics Institute, 2/3 Academichesky Ave, Tomsk, 634055, Russia \\ ${ }^{2}$ Lawrence Berkeley National Laboratory, 1 Cyclotron Road, Berkeley, California, 94720, USA
}

DISCLAIMER

This document was prepared as an account of work sponsored in part by the United States Government. While this document is believed to contain correct information, neither the United States Government nor any agency thereof, nor The Regents of the University of California, nor any of their employees, makes any warranty, express or implied, or assumes any legal responsibility for the accuracy, completeness, or usefulness of any information, apparatus, product, or process disclosed, or represents that its use would not infringe privately owned rights. Reference herein to any specific commercial product, process, or service by its trade name, trademark, manufacturer, or otherwise, does not necessarily constitute or imply its endorsement, recommendation, or favoring by the United States Government or any agency thereof, or The Regents of the University of California. The views and opinions of authors expressed herein do not necessarily state or reflect those of the United States Government or any agency thereof or The Regents of the University of California. 


\title{
Ion Species and Charge States of Vacuum Arc
}

\section{Plasma with Gas Feed and Longitudinal Magnetic}

\author{
Field \\ Efim Oks ${ }^{1}$ and André Anders ${ }^{2}$ \\ ${ }^{1}$ High Current Electronics Institute, 2/3 Academichesky Ave, Tomsk, 634055, Russia \\ 2 Lawrence Berkeley National Laboratory, 1 Cyclotron Road, Berkeley, California, 94720, USA
}

\begin{abstract}
The evolution of copper ion species and charge state distributions is measured for a long vacuum arc discharge plasma operated in the presence of a longitudinal magnetic field of several $10 \mathrm{mT}$ and working gas (Ar). It was found that changing the cathode-anode distance within $20 \mathrm{~cm}$ as well as increasing the gas pressure did not affect the arc burning voltage and power dissipation by much. In contrast, burning voltage and power dissipation were greatly increased as the magnetic field was increased. The longer the discharge gap the greater was the fraction of gaseous ions and the lower the fraction of metal ions, while the mean ion charge state was reduced. It is argued that the results are affected by charge exchange collisions and electron impact ionization.
\end{abstract}

\section{INTRODUCTION}

The term "vacuum arc" suggests that no gaseous ions would appear in this kind of discharge because the main ionization processes take place at cathodic arc spots and close to the cathode region, where plasma is produced from electrode material. Near the cathode, the density of metal plasma is much higher than the density of residual gas even if additional gas was supplied into the discharge gap. This is indeed correct up to the relatively high background pressure of about $10^{-4}$ Torr. No essential ionization of gaseous molecules was observed in experiments [1]. However, it is important to note that an increase of background pressure influenced the metal ion charge state distribution. The presence of gas greatly reduces the higher charge states of metal ions, confirming the rule that in order to obtain the highest charge states, one must decrease the background gas pressure to the minimum possible. There is ample and firm experimental evidence for charge-exchange as the main process responsible for reducing the metal ion mean charge state in the presence of gas neutrals [2-4].

When the vacuum arc with gas feeding is combined

The work was supported by DOE IPP, project LBNL-T2-0196, and with a magnetic field, the charges states have still a tendency to fall, though a large fraction of gaseous ions appears [1,5]. As follows from our previous publication, the ionization of gaseous molecules takes place in the volume of the discharge gap [4].

This paper presents results of more detailed investigations of both processes, namely the reduction of high charge states of metal ions and the appearance of gaseous ions. We conduct the experiments using a vacuum arc discharge ignited in an extended gap with gas feed and in the presence of a longitudinal magnetic field. The work follows and concludes previous research done in close collaboration between the research groups in Berkeley and Tomsk [6].

\section{EXPERIMENTAL SET-UP}

The schematic of the experimental set-up is shown in Fig. 1. It is based on the "MEVVA V" vacuum arc ion source at Lawrence Berkeley National Laboratory [7]. For the present experiments, the cathode unit of the ion source was replaced by a long solenoid of $8 \mathrm{~cm}$ inner diameter and $20 \mathrm{~cm}$ length. A vacuum arc copper cathode (1) with the traditional flashover trigger system was located inside the coil (6). The inner surface of the coil body (5) served also as the vacuum arc anode. The cathode together with the trigger ring (2) and ceramic (3) was fixed onto the top end of a movable stainless steel rod (4). This gave us the possibility to change the location of the cathode unit within the coil from position $L=0$ to $L=20 \mathrm{~cm}$. The zero location was chosen at the coil end facing the MEVVA extraction system.

Working gas argon was fed from the other side of the coil. Because of a rather wide extraction area, the pressure drop between discharge area and the body of the vacuum vessel was relatively small. The traditional "MEVVA V" ion source power supply and its high voltage triggering system was used for operating the vacuum arc. In all experiments the parameters of the arc current remained the same, namely $200 \mathrm{~A}, 300 \mu \mathrm{s}$, 
with 0.3 pulses per second. The coil current was supplied by discharging an assembly of 10 capacitors in parallel with a total capacity of $250 \mu \mathrm{F}$. Triggering of the magnetic field coil current was controlled by a thyristor switch. A relatively low pulse repetition rate was chosen in order to provide enough time between pulses to charge the capacitor for the magnetic coil. Measurements of the pulsed magnetic field by a small probe coil had shown that the B-field reached it maximum at about $5.5 \mathrm{~ms}$ after start of the coil current.
Therefore the arc triggering was delayed by approximately $5.5 \mathrm{~ms}$ to utilize the maximum field. At the maximum of the coil current, the magnetic coil provided approximately $10 \mathrm{mT}$ in the center of the coil per 1 A coil current.

Ions were extracted and accelerated by up to 40 $\mathrm{kV}$ and then analyzed by a time-of-flight (TOF) spectrometer [8]. All measurements of the ion spectra were made at the middle of the arc pulse, i.e., at approximately $150 \mu$ s after arc start.

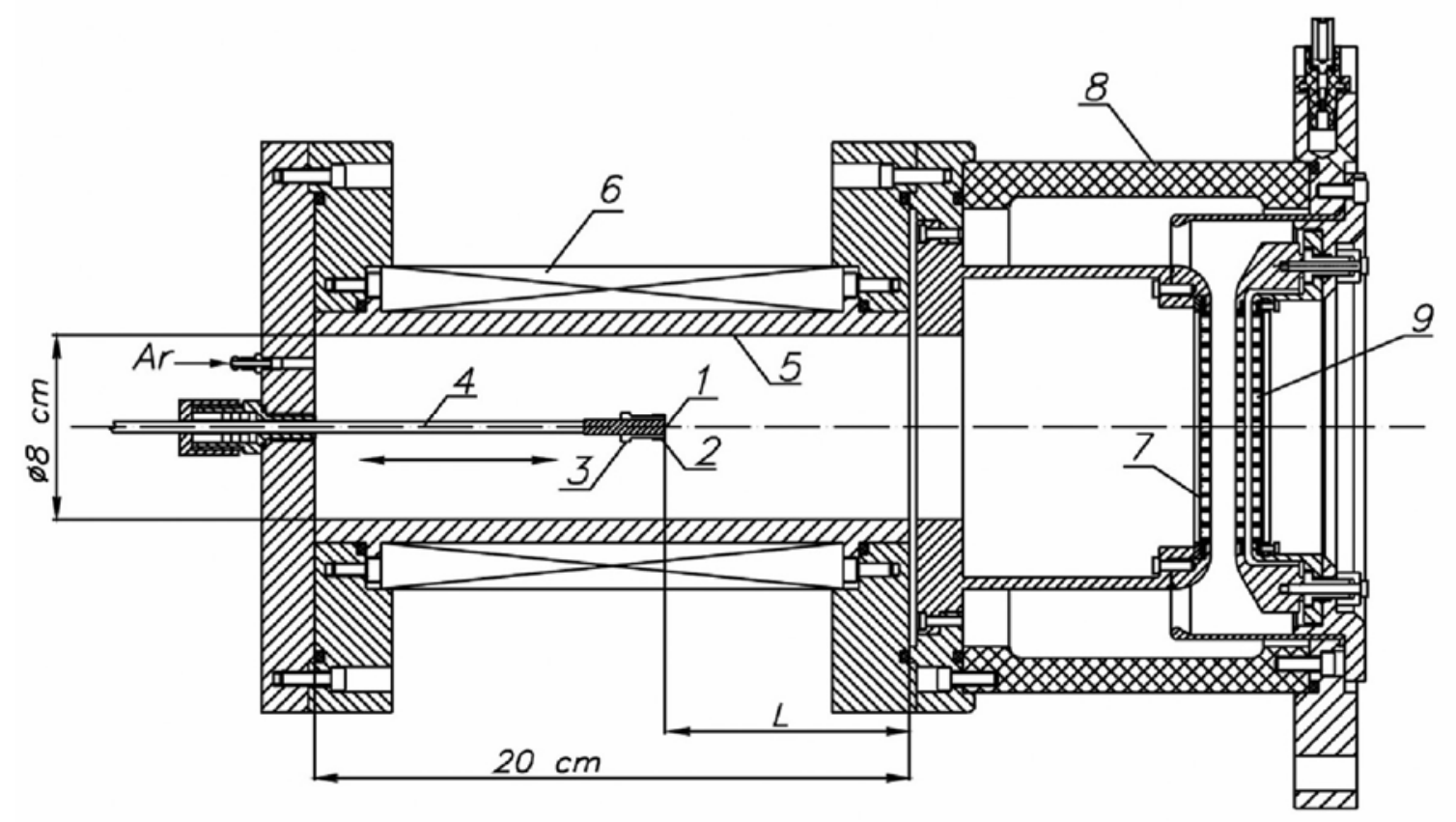

Fig.1. Experimental set-up: 1 - copper cathode, 2 - triggering ring, 3 - ceramic flashover ring, 4 movable stainless steel rod, 5 - anode, 6 - coil, 7 emission (plasma) grid, 8- high voltage insulator, 9 - ion extraction grids.

\section{EXPERIMENTAL RESULTS}

As followed from the experiments, moving the cathode within the $20 \mathrm{~cm}$ distance from the extractor grid did not much influence the arc burning voltage; it changed from $U_{\mathrm{b}}=26 \mathrm{~V}$ at $L=0 \mathrm{~cm}$ to $U_{\mathrm{b}}=24 \mathrm{~V}$ at $L=$ $20 \mathrm{~cm}$. The burning voltage was much more influenced by the magnetic field. Without magnetic field it was 25 $\mathrm{V}$, whereas with magnetic field it rose to $35 \mathrm{~V}(0.05 \mathrm{~T})$, $40 \mathrm{~V}(0.1 \mathrm{~T})$ and $44 \mathrm{~V}(0.2 \mathrm{~T})$. Moving the cathode position in the presence of the magnetic field lead to greater changes of the burning voltage than in the absence of the field, namely from $28 \mathrm{~V}$ to $45 \mathrm{~V}$ for positions $0 \mathrm{~cm}$ and $20 \mathrm{~cm}$, respectively. An increase of gas pressure caused a weak reduction of the burning voltage. For example, increasing the pressure from $7.5 \mathrm{x}$ $10^{-4}$ Torr to $2.4 \times 10^{-4}$ Torr correlated to a decrease from $40 \mathrm{~V}$ to $36 \mathrm{~V}$.

As expected, moving the cathode far away from the extraction area had a significant influence on the extracted ion current. Moving to $L=20 \mathrm{~cm}$, the total ion beam current decreased from $90 \mathrm{~mA}$ to $16 \mathrm{~mA}$.
An increase of the gas pressure caused a relatively minor decrease of the extracted ion current. As follows from the experiments, the ion current fell from $44 \mathrm{~mA}$ to $25 \mathrm{~mA}$ when the pressure of argon increased from $7.5 \mathrm{x}$ $10^{-5}$ Torr to $2.4 \times 10^{-4}$ Torr.

Figs. 2 and 3 show TOF spectra of extracted ion beams for the two extreme cases $L=0 \mathrm{~cm}$ and $L=20$ $\mathrm{cm}$. It is clearly visible that the further away the location of the cathode from the ion extraction zone the more gaseous ions appear in the ion beam. More detailed data of such tendency can be found in Fig. 4, which shows the composition of the extracted ion beam for different cathode positions. Another important conclusion is that the decrease of the high charge state fraction of copper ions corresponds to an increase of the fraction of lower charge states.

Feeding gas into the discharge gap and thus increasing the gas pressure enables the generation of gaseous ions. As follows from the experimental results shown in Fig. 5, the longer the distance between the cathode spots and the location of investigation, given by the extraction system, the more gaseous ions appear in the extracted ion beam. The level of the gaseous ion 
fraction is determined by the gas pressure.

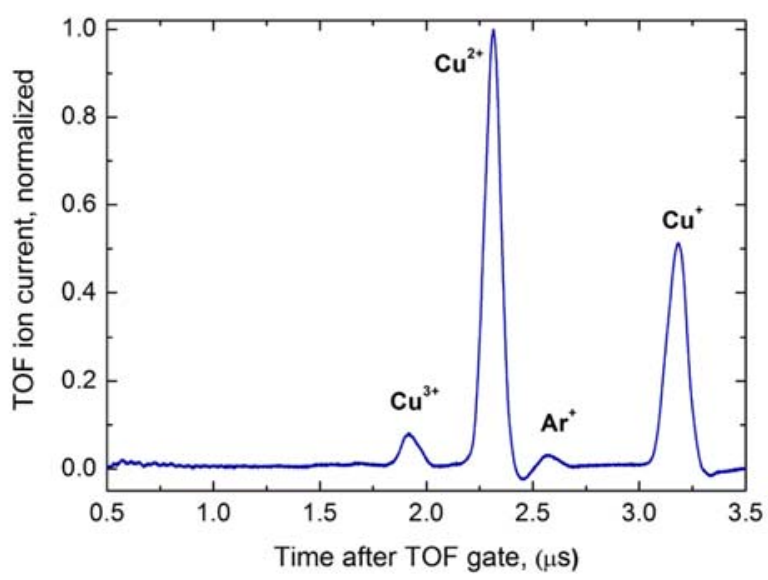

Fig.2. Ion beam mass/charge spectra for cathode position $0 \mathrm{~cm}, B=$ $0.05 \mathrm{~T}$, argon $p=1 \times 10^{-4}$ Torr.

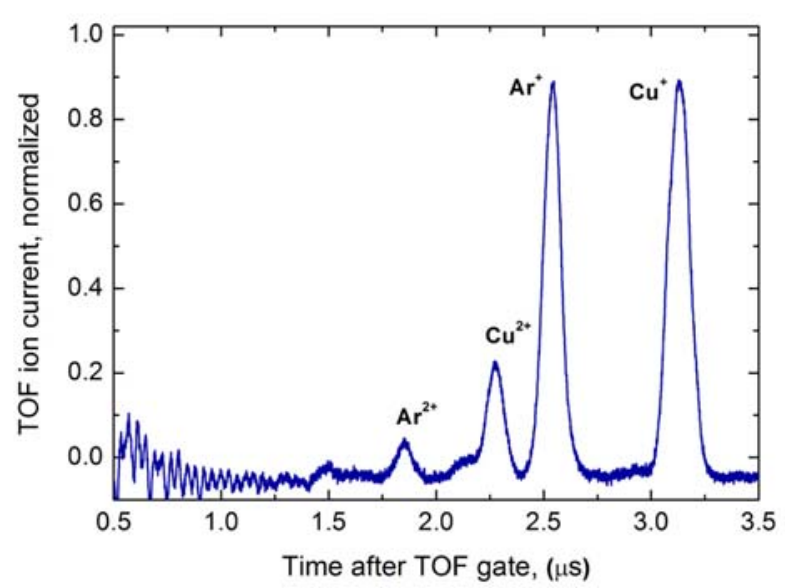

Fig.3. Ion beam mass/charge spectra for cathode position $20 \mathrm{~cm}, B=$ $0.2 \mathrm{~T}$, argon $p=1 \times 10^{-4}$ Torr.

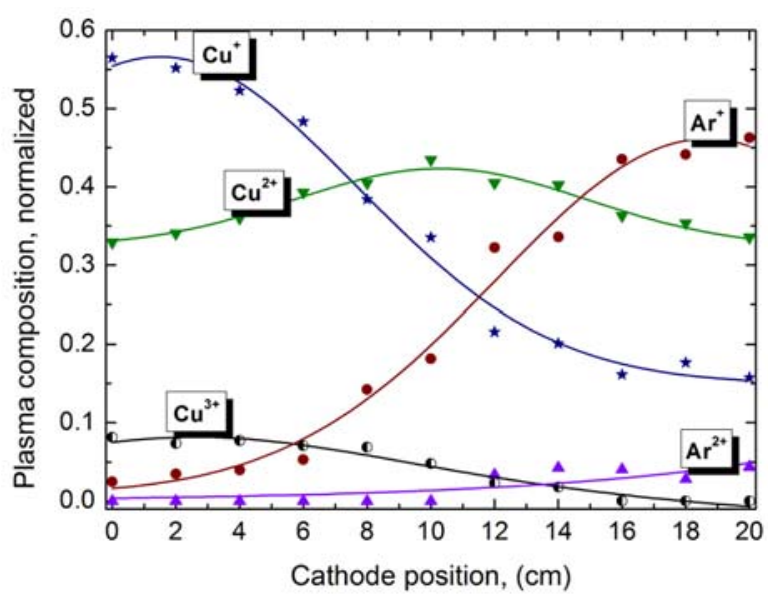

Fig.4. Composition of the extracted ion beam at different cathode positions. Arc current $200 \mathrm{~A}$, argon, $p=7.4 \times 10^{-5}$ Torr, $B=0.2 \mathrm{~T}$.

The mean charge state of the metal ions also depends on the discharge gap distance. The pressure is critically important for the reduction of the high charge state fractions of metal ions (Fig.6). The higher the pressure the stronger the influence of the cathode position on the charge composition of the metal ions.

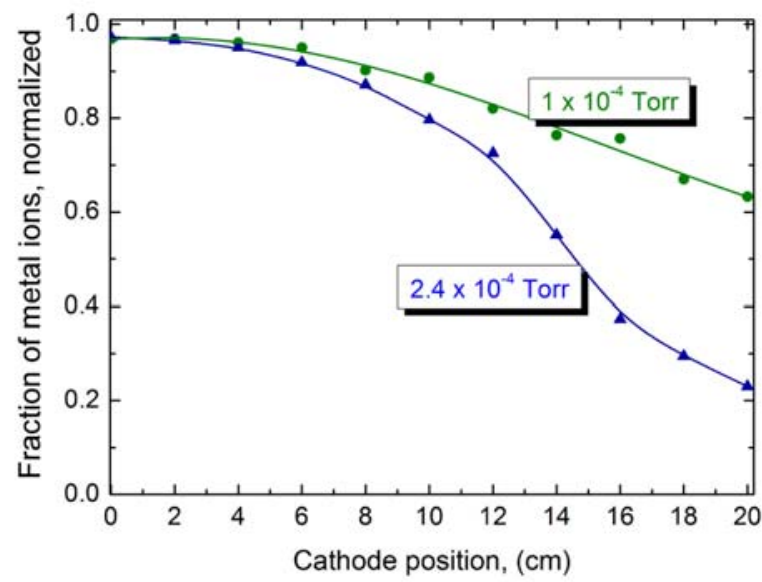

Fig.5. Total fraction of metal (copper) ions in extracted beam at different cathode positions. Arc current $200 \mathrm{~A}, B=0.2 \mathrm{~T}$.

We can summarize the findings as follows. Feeding gas into the discharge gap leads to two effects. First, the gas decreases the high ion charge state and correspondingly increases the low charge states. Second, the gas also enables the generation of gaseous ions.

For the first effect, only the gas pressure is important. In contrast, the appearance of gaseous ions is greatly affected by the presence of the magnetic field, as clearly illustrated by the experimental results of Fig. 7 . Without magnetic field, only small amounts of gaseous ions can be found provided the cathode is positioned at a rather long distance to the extraction zone. The situation changes dramatically when even only a rather weak magnetic field is applied.

\section{DISCUSSION}

This distinct behavior can be explained taking the different types of collisions into account and whether or not free electrons are involved in them.

The reduction of high metal ion charge states and the corresponding increase of lower metal ion charge states is a "fingerprint" of charge exchange collisions. When higher charged metal ions collide with metal neutrals, charge transfer reduces the higher charge state by one, and a singly charged metal ion appears. The gas atoms themselves can be a collision partner, though ionization of argon is unlikely due to the energy defect [2-4]; it is only likely in three particle collisions involving a doubly charged copper ion. Gas atoms can slow down copper ions by elastic collisions, and thereby promote the formation of copper neutrals near walls [2-4], which in turn affects the charge exchange rate. The presence of the magnetic field does not affect the motion of atoms, and only marginally those of ions, and hence charge exchange collisions are not very sensitive 
to the presence of the field.

In contrast, the appearance of gaseous ions is caused by electron impact ionization. Free electrons are greatly affected by the magnetic field because their gyration radius is much smaller than the characteristic system size (here the solenoid radius). Therefore it does not surprise that the likelihood of ionization of the background gas is enhanced as soon as the magnetic field is switched on. Higher gas pressure also promotes an increased likelihood of electron-gas elastic collisions, which leads to cooling of the electrons, followed by a reduced ability to cause ionization.

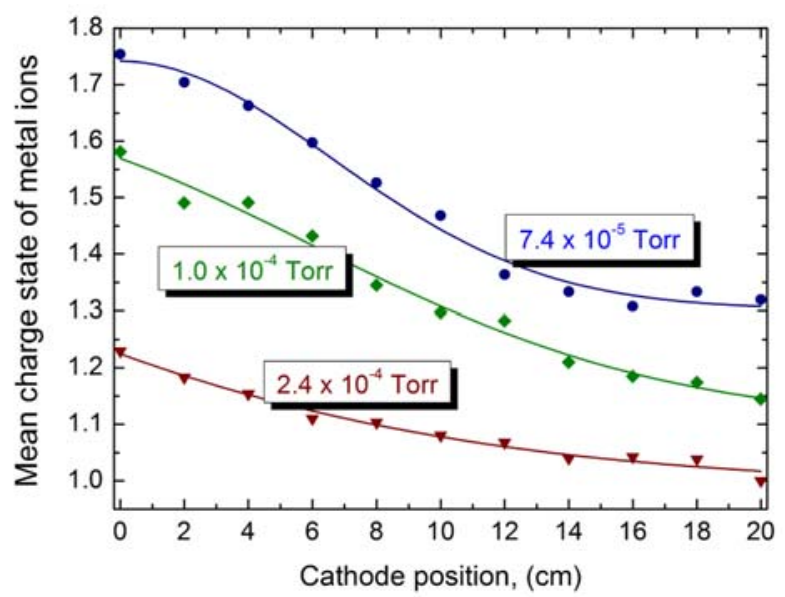

Fig. 6. Metal (copper) ion mean charge state in extracted beam at different cathode position. Arc current $200 \mathrm{~A}, B=0.2 \mathrm{~T}$.

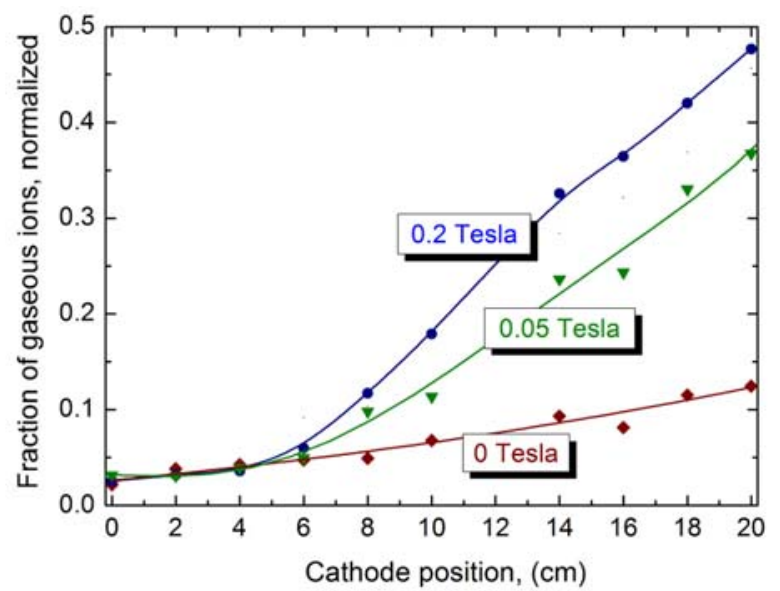

Fig. 7. Total fraction of gaseous ions (Ar $p=1.0 \times 10^{-4}$ Torr) in the extracted beam for different cathode positions. Arc current $200 \mathrm{~A}$.

\section{CONCLUSIONS}

The experimental results presented in this paper are complimentary to our previous findings that the processes of gas ionization and reduction of metal ion mean charge state take place not at or near the cathode surface but in the space between the cathode and anode. Different collision processes with neutral atoms are responsible for this. They include, but are not limited to, (i) charge exchange of multiply charged metal ions with metal neutrals, and (ii) gas ionization by the energetic electrons of the tail of the Maxwell distribution. The presence of gas affects both types of collisions. Since electrons are readily magnetized, collision types involving free electrons are very sensitive to the absence or presence of a magnetic field.

We hope that the results of these experiments will promote the creation of more detailed physical models of the cathodic arc plasma and its interaction with gas atoms and external fields. The results have also relevance to the cathode processes themselves, for example they imply that the charge states of metal ions at cathode spots are higher than generally observed in some distance because the observed values resulted from plasma that had interacted with gas or the metal neutrals [2,9].

\section{ACKNOWLEDGMENTS}

We are grateful to Dr. Georgy Yushkov, HCEI Tomsk, for his suggestion of such experimental research, and fruitful discussions.

\section{REFERENCES}

[1] P. Spädtke, et al., "Influence of gas added to the MEVVA discharge on the extracted ion beam,” Rev. Sci. Instrum., vol. 65, pp. 3113-3118, 1994.

[2] A. Anders, E.M Oks and G.Yu. Yushkov, "Production of neutrals and their and effects on the ion charge states in cathodic vacuum arc plasmas," J. Appl. Phys., vol. 102, pp. 043303/1-7, 2007.

[3] A. Anders and G. Yu Yushkov, "Puzzling differences in bismuth and lead plasmas: Evidence for the significant role of neutrals in cathodic vacuum arcs," Appl. Phys. Lett., vol. 91, pp. 091502/1-3, 2007.

[4] E.M Oks, G.Yu Yushkov and A. Anders, “Temporal development of ion beam mean charge state in pulsed vacuum arc ion sources," Rev. Sci. Instrum., vol.79, pp. 02B301/1-3, 2008.

[5] B.H. Wolf, et al., "A Mevva ion source for simultaneous implantation of gas and metal ions,” Nucl. Instrum. Meth. Phys. Res. B, vol. 106, pp. 417-428, 1995.

[6] See various papers of A. Anders, I.G. Brown, E.M. Oks, G.Yu. Yushkov and others co-authors in previous Proceedings of the ISDEIV (1996-2008).

[7] I.G. Brown, "Vacuum arc ion sources," Rev. Sci. Instrum., vol. 65, pp. 3061-3082, 1994.

[8] V.I. Gushenets, et al., "Simple and inexpensive time-of-flight charge-to-mass analyzer for ion beam source characterization," Rev. Sci. Instrum., vol. 77(b), pp. 063301, 2006.

[9] A. Anders, Cathodic Arcs, Springer, New York, 2008.

\section{E-mail of authors:}

oks@opee.hcei.tsc.ru and aanders@lbl.gov 